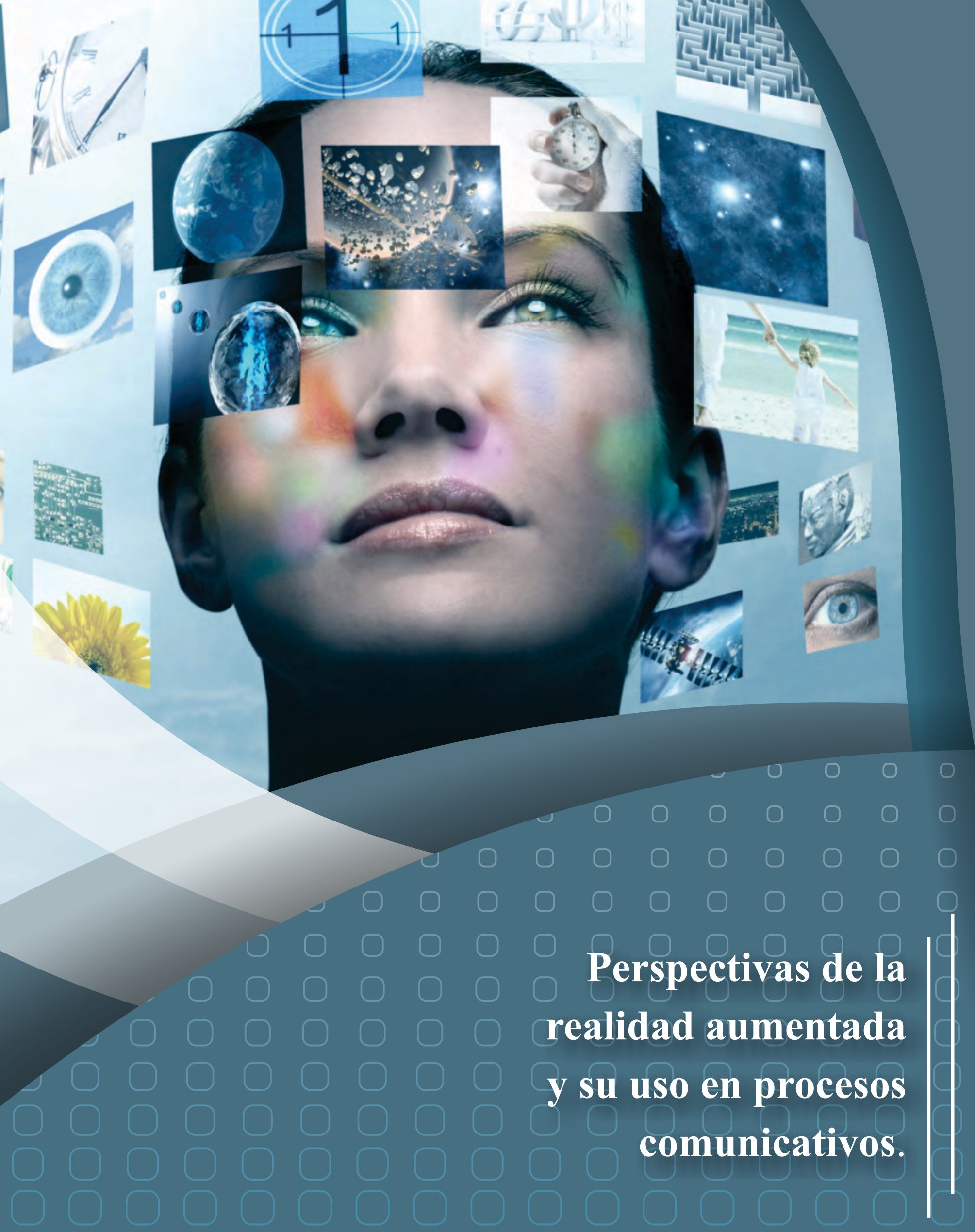




\title{
Perspectivas de la realidad aumentada y su uso en procesos comunicativos. ${ }^{1}$
}

\section{Augmented Reality Perspectives and its applications in Communication Processes.}

\author{
Jorge Antonio Alvarado Rojas ${ }^{2}$, Carlos Gustavo Román Echeverri ${ }^{3}$ \\ ${ }^{1}$ Fundación Universitaria San Martín, Colombia.
}

\begin{abstract}
Artículo recibido en el mes de Julio de 2013; artículo aceptado en el mes de Octubre de 2013.
\end{abstract}
Citación del artículo: Alvarado, R. \& Román, C. (2013). Perspectivas de la realidad aumentada y su uso en procesos comunicativos. I+D Revista de Investigaciones, 2(2), 76-88.

\section{Resumen}

La tecnología de la Realidad Aumentada derivada del área de generación de ambientes virtuales - consiste en el despliegue de elementos virtuales que complementan el entorno físico real a través de dispositivos de captura y reproducción de video, sonido y/o animación digital. Las técnicas de realidad aumentada han sido empleadas en diversas aplicaciones (comerciales, educativas, científicas, artísticas) conformando un canal híbrido de comunicación que se utiliza para potenciar la realidad del espectador. El uso de la realidad aumentada implica cambios significativos en los procesos de comunicación multimediáticos, ya que expande sus posibilidades dentro de un entorno físico finito, descentralizando los canales tradicionales y proporcionando un entorno interactivo, en el que el rol del usuario cobra mayor importancia y ayuda a definir la experiencia. En el texto se analizan las ventajas y desventajas del uso de la realidad aumentada como herramienta comunicativa, explorando el potencial de este tipo de tecnologías y revisando ejemplos concretos del contexto local e internacional.

Palabras clave: Realidad Aumentada, multimedia, comunicación digital, ambientes virtuales.

\footnotetext{
${ }^{1}$ Artículo de reflexión, de enfoque cualitativo, resultado de un proyecto de investigación. Desarrollado en el grupo de investigación Nimbus de la Fundación Universitaria San Martín (Colombia).

${ }^{2}$ Ingeniero de Sistemas, Universidad Pedagógica y Tecnológica de Colombia. Especialista en creación multimedia, Universidad de los Andes de Bogotá. Docente-investigador del grupo Desarrollo de Ambientes Virtuales. Fundación Universitaria San Martín(Colombia): Correo-e: jorge.alvarado@ingenieria.sanmartin.edu.co

${ }^{3}$ Ingeniero de Sistemas, Universidad Pedagógica y Tecnológica de Colombia. Especialista en creación multimedia, Universidad de los Andes de Bogotá. Docente-investigador del grupo Nimbus. Fundación Universitaria San Martín (Colombia): Correo: carlos.roman@ingenieria.sanmartin.edu.co
} 


\section{Abstract}

The Augmented Reality Technology - derived from the creation of virtual environmentsconsists in the virtual elements displaying, complementary to the physical real environment by means of devices for capturing and reproduction of video, sound and/or digital animation. The augmented reality techniques have been used in several applications (commercial, educational, scientific, artistical) constituting an hybrid communication channel for making more powerful the reality to the spectator. The using of augmented reality implies meaningful changes in multimedia communication processes because of expanding its possibilities in a finite physical environment, decentralizing the usual channels and providing an interactive context where the user's role becomes into important and helps in the experience definition. In the text the advantages and disadvantages are analyzed in the using of augmented reality as a communication tool, exploring the potential of these technologies and reviewing specific examples in the local and international context.

Keywords: Augmented reality, multimedia, digital communication, virtual environments.

\section{Introducción}

Al repensar la producción de imágenes en la contemporaneidad bajo el lente de los cambios socio-culturales introducidos por los avances en las tecnologías de la información y las comunicaciones de los últimos años, Manovich (2006) plantea algunos aspectos relevantes que deben ser considerados para plantear un panorama preliminar del problema: el surgimiento de una sociedad global configurada a través de redes; la complejidad creciente de los sistemas de información y la disponibilidad de la misma en tiempo real; la multiplicidad y naturaleza diversa de las fuentes; la necesidad de representar cada vez más datos en soportes novedosos; la fragmentación de sectores económicos centralizados en el consumidor. En este contexto, Manovich no propone la creación de imágenes totalmente nuevas sin antecedentes históricos, sino la adaptación de imágenes existentes mediante la adición de mecanismos de interactividad, navegabilidad y manipulabilidad, a través de las técnicas que han permitido la computarización de la cultura visual en las últimas décadas.

Precisamente, la generación de entornos virtuales corresponde a esta coyuntura compleja y responde a estos parámetros específicos de adaptabilidad. El campo de la realidad virtual se ha consolidado entonces como un vehículo de representación del mundo a través de la generación de imágenes de diversa índole sensorial (visuales, sonoras, táctiles, etc.) que tratan de recrear la tridimensionalidad del mundo real, creadas por medios computacionales y desplegadas a través de dispositivos de salida como pantallas, parlantes o superficies. Pero mientras la Realidad Virtual trata de crear universos completamente concebidos por computador, el campo de la Realidad Mixta se dedica a combinar elementos del mundo real con elementos virtuales, subdividiéndose en dos áreas: 1a Virtualidad Aumentada (una combinación que presenta más elementos digitales que reales) y la Realidad Aumentada (se 
aplica a los ambientes en donde la cantidad de elementos digitales representados corresponden en un porcentaje inferior al de los elementos presentados del ambiente real). La Realidad Aumentada (RA) trata de enriquecer los mecanismos de percepción del mundo real del usuario con objetos informativos generados por un computador, de tal forma que dicha información se encuentre relacionada con elementos del entorno y logre complementar y enriquecer - es decir, 'aumentar' - la experiencia. En el contexto del proyecto "Caracterización de metodologías, herramientas y aplicaciones en el desarrollo de ambientes virtuales basados en técnicas de Realidad Aumentada" realizado por la línea de interés Desarrollo de ambientes virtuales del grupo de investigación Nimbus (Colciencias) de la Fundación Universitaria San Martín, se busca en primera instancia analizar el rol de las tecnologías de RA en procesos de comunicación (personal y/o masiva) contemporáneos, por lo cual en este artículo se siguió un tipo de estudio documental e interpretativo, acerca de un fenómeno del cual se hacen reflexiones de sus supuestos, contenidos, estructura $\mathrm{y}$ funcionamiento, para derivar en conclusiones sobre el mismo, y se reflexiona sobre el estado del arte en el tema, tratando de clasificar las aplicaciones más notables y encontrando las tendencias más relevantes en el campo. De igual manera, se revisan las ventajas y desventajas del uso de la RA como herramienta comunicativa, apoyados en ejemplos concretos del contexto local e internacional y explorando posibles perspectivas de aplicabilidad en un futuro cercano.

\section{Desarrollo}

\section{Antecedentes históricos.}

En el campo de la generación de entornos virtuales, se considera como pionero el trabajo de Ivan Shuterland (1968), llamado La espada de Damocles, en el que se disponía de un dispositivo de visualización similar a un casco (en inglés: Head-Mounted Display) para lograr una experiencia de inmersión en un ambiente simulado de tres dimensiones (Zhou, Duh \& Billinghurst, 2010). Este proyecto ya contenía características de lo que años más tarde iba a llamarse Realidad Mixta, como lo es la superposición de imágenes computarizadas dentro del campo de visión natural del usuario. El desarrollo conceptual y técnico de la realidad virtual continuaría en los años siguientes, hasta que en la década del noventa empieza a aparecer con mayor frecuencia el término Realidad Aumentada de manera formal en trabajos investigativos. Azuma (1997) establece un esquema estructurado sobre las características de las entornos de RA y a finales de la década del noventa aparecen también los primeros simposios internacionales sobre el tema, como por ejemplo el International Workshop and Symposium on Augmented Reality (IWAR), el International Symposium on Mixed Reality (ISMR), el International Symposium on Augmented Reality (ISAR) o el Designing Augmented Reality Environments Workshop (Zhou, Duh \& Billinghurst,2010). En la última década, los usos, técnicas y campos de acción de la RA se han diversificado enormemente, conformándose el International Symposium on 
Mixed and Augmented Reality (ISMAR) como uno de los espacios académicos globales más relevantes que trata de abarcar la temática en la actualidad.

\section{Aspectos definitorios de la realidad aumentada.}

Para Azuma et al. (2001) una aplicación es considerada y catalogada como perteneciente al campo de la RA, si posee tres características esenciales, a saber: (a) combinación de elementos reales y virtuales, (b) interactividad en tiempo real, (c) registro y alineación de los objetos reales y virtuales entre sí.

Una serie de dispositivos y procesos tecnológicos específicos se requiere para lograr desarrollar una aplicación de RA. A continuación se especifican aquellos más relevantes de la estructura general, organizados en: (a) Dispositivos de visualización, (b) técnicas de rastreo, (c) calibración e interacción, y (d) herramientas comunes.

\section{(a) dispositivos de visualización.}

En esta categoría se agrupan todos los elementos que permiten proyectar los entornos aumentados para ser percibidos a través de los mecanismos de visión. Dentro de los más empleados según Zhou, Duh y Billinghurst (2010), se encuentran los dispositivos de visualización que se usan en la cabeza (en inglés: head-worn display), que pueden ser cascos o gafas que usan pequeñas pantallas con espejos que reflejan las imágenes sobre los lentes, o pantallas translúcidas que permiten observar el ambiente real a la vez que se sobreponen los datos generados por el computador. También se pueden utilizar dispositivos de visualización portátiles o manuales (en inglés: handheld display) que tienen una cámara incluida que graba el entorno real, generando y combinando la información para que el usuario la vea en una pantalla plana portable. En esta última categoría se incluyen los dispositivos móviles como teléfonos celulares, como el que se muestra en la figura 1, o las tablets, cuyo uso actualmente se está masificando.

Figura 1. Aplicación de RA desde un dispositivo móvil.

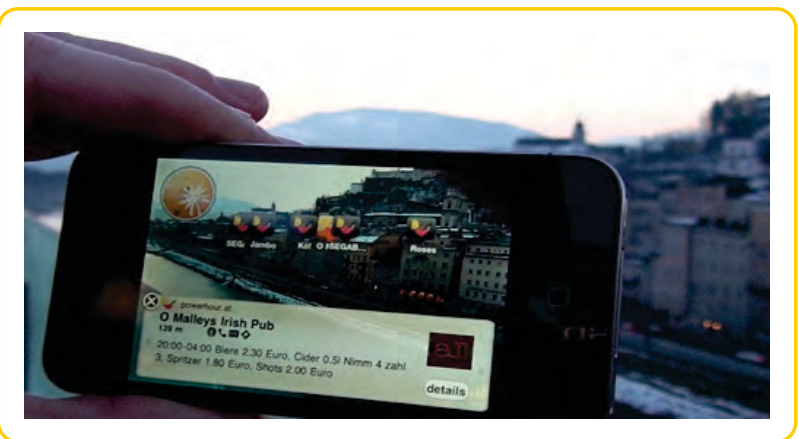

Fuente: http://commons.wikimedia.org/wiki/ File:Wikitude_World_Browser_@Salzburg_Ol d_Town_3.jpg. Reproducida con autorización por licencia Creative Commons.

Una tercera categoría de dispositivos de visualización son los dispositivos de visualización de proyección (en inglés: projection displays), que permiten combinar los elementos virtuales sobre el espacio real $\mathrm{u}$ ocultar elementos del espacio real; esto se hace cubriendo estos objetos con material reflectivo y proyectando sobre ellos imágenes procesadas digitalmente (Azuma, 2001).

\section{(b) técnicas de rastreo.}

Una de las técnicas más comunes que se emplean en aplicaciones de RA es el rastreo, que consiste en detectar algún tipo de imagen o 
patrón sobre el cual se generará el contenido virtual aumentado. En algunos casos se usan marcadores, que son pequeñas imágenes con patrones gráficos predefinidos, diseñados para ser procesados e identificados por el computador dependiendo de su lugar, posición y rotación. En otros casos se usan sensores de posicionamiento que permiten aumentar el ambiente con información u objetos construidos en 3D (Azuma, 2001; Zhou, Duh \& Billinghurst, 2010). Para el rastreo en ambientes al aire libre o aplicaciones móviles, no es práctico cubrir todo el espacio con marcadores, de forma que se utiliza rastreo a través de video apoyado con el uso de dispositivos de localización geográfica como brújulas y giroscopios; el rastreo de patrones en ambientes no preparados también se puede hacer mediante el reconocimiento de figuras y formas tanto naturales como artificiales del ambiente, así como la aplicación mostrada en la figura 2, que al reconocer una pintura específica proporciona información adicional sobre la misma.

Figura 2. Reconocimiento de patrones en objetos existentes dentro un entorno no preparado.

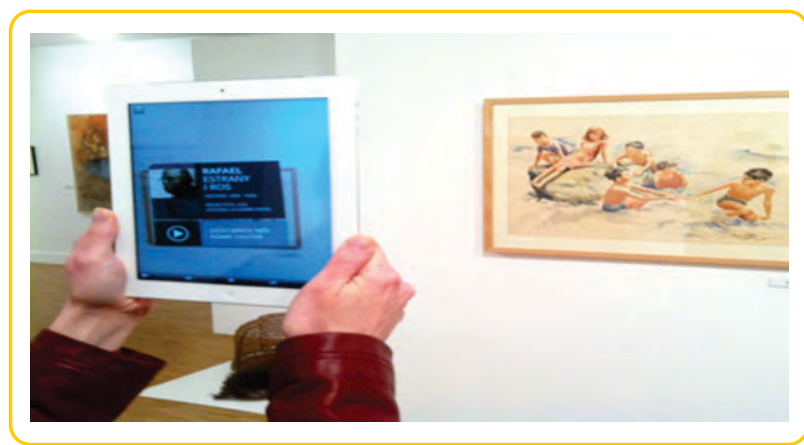

Fuente: http://commons.wikimedia.org/wiki/File:

Augmented_reality_at_Museu_de_Matar\%C3\%B3_linki ng_to_Catalan_Wikipedia_(29).JPG Reproducida con autorización por licencia Creative Commons

\section{(c) calibración.}

Los sistemas de RA necesitan ejecutar una calibración del entorno, para hacer un registro preciso de los elementos virtuales en el espacio real, es decir, posicionar los elementos generados por computador de manera que sean coherentes con aquellos del entorno real. Se puede realizar el proceso de dos maneras: calibración manual, realizada por el usuario; o calibración automática que se realiza mediante revisión y realimentación de puntos de referencia ubicados en el ambiente real.

\section{(d) técnicas de interacción e interfaces de}

\section{usuario.}

Existen técnicas de interacción que se usan según los requerimientos de cada aplicación. Cuando se usan elementos reales como marcadores o sensores que pueden ser manipulados por el usuario para controlar los elementos virtuales, se conoce como una RA Tangible (Zhou, Duh \& Billinghurst, 2010). Esta técnica es bastante utilizada, porque provee una manera más intuitiva de interactuar con los objetos virtuales mediante la manipulación de objetos en el ambiente real. Aunque en términos generales pareciera que las aplicaciones de RA fueran concebidas para experiencias individuales de tipo mono-usuario, existe otra clase de interfaces de tipo colaborativo, en donde los usuarios viven una experiencia compartida por medio de su interacción con otros usuarios, que puede ser de manera presencial con dispositivos de visualización manuales, o de manera remota como en aplicaciones de RA para teleconferencias (Zhou, Duh \& Billinghurst, 2010). 
Este tipo de interfaces de tipo colaborativo son mucho más intuitivas y se apoyan en los protocolos sociales definidos por los usuarios. Un tercer tipo de interacción que se puede definir para las interfaces de RA es el híbrido, en donde se confinan distintas formas de interacción (tangibles o sobre dispositivos de visualización manuales) con diversas formas de visualización (Zhou, Duh \& Billinghurst, 2010).

Las llamadas interfaces hápticas también son utilizadas en la interacción con aplicaciones de RA. Este tipo de interfaces (que pueden ser robots en algunas ocasiones) interactúan con los usuarios, realimentando sus acciones con respuestas físicas que emulan los efectos de los objetos virtuales a través de estímulos motores y táctiles. Los guantes de datos permiten a los usuarios manipular objetos virtuales en las aplicaciones de RA y rastrear los movimientos de las manos, aspecto que puede entorpecer la movilidad en el espacio real. Hay otras tecnologías emergentes en el campo de las interfaces de usuario para aplicaciones de RA como el rastreo de movimientos oculares, corporales y el reconocimiento de voz (van Krevelen \& Poelman,2010).

\section{(d) herramientas.}

Las aplicaciones de RA trabajan sobre el reconocimiento del espacio tridimensional, por lo que resulta necesario en muchas ocasiones emplear herramientas de modelado en 3D. Por ejemplo, el lenguaje VRML (Virtual Reality Modeling Language) es empleado para modelar objetos digitales en tres dimensiones, junto con librerías que ayudan a realizar el proceso de renderizado de estos objetos en tiempo real. Existen infraestructuras digitales (o frameworks en inglés) para desarrollo de aplicaciones de RA, siendo ARToolKit una de las pioneras en proponer el uso de marcadores para visualizar elementos virtuales en entornos reales (Kesim \& Ozarslan, 2012). Aunque ésta sea una de la más conocidas y utilizadas, existen otras infraestructuras digitales como StudierStube, DWARF, D'Fusion y el navegador para teléfonos móviles Layar (Kato \& Billinghurst, 1999)

\section{Aplicaciones de la Realidad Aumentada}

La RA, dadas sus características técnicas, ha sido empleada desde sus inicios con fines prácticos en una diversidad de aplicaciones. A continuación se enumeran algunas de ellas.

\section{(a) educación}

La RA ha sido utilizada en procesos educativos por medio de formas diversas de interacción, permitiendo en algunas ocasiones repensar los libros tradicionales para que incluyan aplicaciones de este tipo que ayuden a las personas con poco conocimiento del uso de los computadores a tener una experiencia interactiva (Basogain, Olabe, Espinosa \& Rouèche, 2006). Un ejemplo es el del proyecto MagicBook (Carracedo, 2011) donde las páginas de un libro, al ser vistas por medio de un dispositivo de visualización manual, proyecta imágenes en tres dimensiones que le permiten al usuario comprender mejor el tema de estudio. El Massachusetts Institute of Technology (MIT) es una de las instituciones educativas que ha desarrollado juegos educativos de interior $y$ exterior que usan RA. Estos proyectos de aplicaciones educativas no se limitan a un sólo nivel de formación sino que abarcan todos los espacios educativos incluyendo el de la educación superior (Basogain, Olabe, Espinosa 
\& Rouèche, s.f.; Carracedo, 2011). En el contexto colombiano, por ejemplo, se ha empleado para visualizar gráficos matemáticos tridimensionales con los cuales el usuario puede interactuar mediante la manipulación de parámetros (Esteban, Restrepo, Trefftz, Jaramillo \& Alvarez, s.f.).

\section{(b)entretenimiento}

Las aplicaciones de realidad aumentada en el campo de los videojuegos son numerosas y entre ellas se pueden encontrar juegos para consolas portátiles, teléfonos móviles $\mathrm{u}$ organizadores personales (Personal Data Administrator, PDA) como Tennis RA, Bolos RA para espacios cerrados y simuladores de batalla con enemigos virtuales para espacios abiertos (van Krevelen \& Poelman, 2010). En el campo de trasmisión de contenidos audiovisuales para entretenimiento, la RA se aplica en los contenidos de programas de competencias deportivas donde se presenta a los espectadores información adicional generada por computador, que se mezcla con las imágenes del mundo real captadas por las cámaras de video. Por ejemplo, en las competencias automovilísticas se visualiza la información de cada conductor con punteros que están alineados en tiempo real con la posición de los automóviles (van Krevelen \& Poelman, 2010).

\section{(c) arte.}

La RA ayuda a potenciar formas diferentes de interacción en instalaciones artísticas. "Hidden Words" es una instalación interactiva que permite ver a un grupo de entre 2 a 6 usuarios, las palabras de sus interlocutores por medio de unos dispositivos de visualización usados en la cabeza, que tienen micrófonos incluidos para registrar al usuario que habla y el volumen de sus palabras, para registrarlas en un ambiente virtual donde se mezclan sobre una mesa ubicada en el espacio real a manera de sombras tridimensionales. Otra instalación similar es "RE-MARK" que convierte las palabras de los usuarios (capturadas con un micrófono) en fonemas que se proyectan en frente del usuario. Una instalación que tiene un mayor nivel de complejidad es "Messa di Voce", contiene varios módulos que pueden ser manipulados por los artistas de forma colaborativa o en solitario; la aplicación registra la posición de los intérpretes y la intensidad de la voz y proyecta figuras que a su vez se proyectan sobre los actores en escena, mezclando así las imágenes digitales con el ambiente real (Levin \& Lieberman, 2004).

\section{(d) medicina.}

Diversos campos específicos de aplicación de la RA en el área de la medicina son mencionados por Ortiz (2011), como el análisis de imágenes biomédicas, la simulación de sistemas fisiológicos, el entrenamiento médico en temas de anatomía, la visualización de procedimientos quirúrgicos o la creación de mapas tridimensionales.

\section{(e) urbanismo.}

Existen aplicaciones como ARTHUR, que permite trabajar entornos urbanos, con el fin de planificar espacios de un barrio y una ciudad, para corregir posibles fallos en la concepción de proyectos urbanísticos por medio de maquetas virtuales que se pueden construir y modificar en tiempo real, de forma colaborativa (van Krevelen \& Poelman, 2010).

\section{(f) turismo y publicidad.}

En el ámbito comercial, se pueden contar 
aplicaciones desarrolladas para publicidad que utilizan marcadores incluidos en impresos y audiovisuales, que se enriquecen con elementos virtuales. De igual forma, existen aplicaciones para móviles que informan a los turistas sobre su entorno y le ofrecen datos sobre sitios de interés, además de referencias históricas de los lugares que visita (van Krevelen \& Poelman,2010).

\section{Realidad Aumentada en procesos de comunicación.}

Una vez revisadas las características principales y aplicaciones más comunes de la RA, cabe preguntarse por su relevancia y grado de afectación en procesos comunicativos clásicos, así como su uso actual en medios masivos de comunicación.

\section{Percepción del entorno}

Las diversas aplicaciones de RA revisadas, emplean un amplio conjunto de elementos de comunicación no verbal, en especial en lo referente a las diversas maneras en las que se puede codificar y descodificar el espacio. El espacio virtual, concebido como imagen $\mathrm{y}$ modelo, no establece una realidad sustancial, sino que cobra sentido como un dispositivo de mediación (Quéau, 1992). La percepción del entorno, según teorías de ambientes de interacción (Knapp, 1982), puede producir reacciones de excitación (grado de actividad y estimulación), bienestar (satisfacción) y seguridad (control y libertad de actuación) en las personas. La RA puede potenciar estas reacciones mediante la inclusión de elementos virtuales con algún propósito comunicativo. En la cotidianidad, el número de entornos espaciales en los cuales se desenvuelven e interactúan las personas, es limitado; incluso en las grandes áreas metropolitanas, el desplazamiento se da a través de circuitos predefinidos entre espacios concretos funcionales (hogar, lugar de trabajo o estudio, sitio de recreación, etc). Las técnicas de RA podrían jugar en este caso un papel importante: aquel de redefinir la experiencia en la percepción de los entornos cotidianos, mediante la adición de información relevante que no esté disponible a nivel sensorial para los seres humanos. De igual manera, aquellos entornos que no presentan un grado de familiaridad muy alto, pueden ser reinterpretados y asimilados de manera positiva. Por ejemplo, las diversas aplicaciones que emplean el Sistema de Posicionamiento Global (en inglés: Global Positioning System, o GPS) una red de satélites que abarca la totalidad de la superficie de la Tierra - se convierten en un canal de comunicación con el que un entorno extraño y desconocido que puede producir sensaciones de inseguridad, tiene el potencial de convertirse en un entorno amable y seguro, mediante la asignación de información geográfica que puede complementarse a través de redes sociales con los comentarios y contenidos generados por los usuarios. La institución financiera colombiana Davivienda $($, por ejemplo, implementó un servicio de RA de ubicación de cajeros y oficinas según la posición local del usuario, brindando información sobre los mismos en tiempo real, permitiendo además realimentar mediante un mecanismo de calificación.

\section{Mediación, figuración y abstracción.}

Para Philippe Quéau (1992), la mediación entre un sujeto y los entornos virtuales, se da principalmente a través de imágenes y modelos. Las imágenes proponen una representación de orden sensorial, y los modelos una representa- 
ción de orden racional. La RA propone entonces una dimensión adicional, cuando se sobreponen elementos virtuales en entornos reales, llegando a conformar un canal híbrido que parte de la intuición en la comprensión y percepción del espacio físico real y se complementa con este dualismo de lo sensible y lo inteligible de los elementos virtuales. Este conjunto de imágenes mediado por la RA - tanto de origen real como virtual -, se debate siempre entre el par antitético figuración/abstracción. Manovich (2006) propone que las herramientas representacionales del mundo digital deberían propender por las imágenes abstractas, en contraposición a la cruzada fotorrealista y figurativa de la realidad virtual. La efectividad de las aplicaciones de RA no se puede medir por su capacidad de representar un mundo virtual como una extensión completamente figurativa del mundo real. Aunque es claro que existen aplicaciones que de alguna manera precisan de una representación fidedigna en los objetos virtuales generados - como aquellas orientadas a la planeación urbanística o la medicina -, existen otras que abren el campo de la RA a la representación abstracta, como es el caso de las instalaciones artísticas o aplicaciones de entretenimiento.

Lo no figurativo puede ser usado en aplicaciones que requieran representaciones de información precisas a través de abstracciones visuales, utilizando figuras convencionales que representen elementos o eventos precisos. Esto permitiría que dichos elementos abstractos aumenten el espacio sin interferir visualmente con la percepción del entorno real, estableciendo una clara diferencia entre las dos fuentes de origen de las imágenes. Una absoluta representación figurativa podría ser contraproducente, ya que, además de implicar mayores costos técnicos, puede hacer que la línea divisoria entre lo virtual y lo real se desvanezca, y lleve al usuario a una apreciación empobrecida de ese mundo aumentado.

\section{Realidad Aumentada y medios masivos de comunicación en el contexto colombiano.}

Varios medios nacionales de prensa escrita han incursionado en el campo de la RA mediante la inclusión de marcadores en sus páginas que, al ser registradas y reconocidas por dispositivos de captura y software de detección, ofrecen información multimedia relacionada $\mathrm{y}$ complementaria con los artículos originales. La revista $\mathrm{SoHo}{ }^{\circledR}$ de Publicaciones Semana S.A.C fue una de las pioneras en el uso de la realidad aumentada en el año 2009, al diseñar una portada que 'cobraba vida' al ser visualizada con un dispositivo de RA. También se incluyen dentro de la revista imágenes publicitarias que con el mismo soporte tecnológico mostraban animaciones y demás contenidos digitales diseñados específicamente para esta plataforma. El diario El Tiempo y El Colombiano también incursionaron en el 2012 con proyectos de Realidad Aumentada. Con estas aplicaciones de RA, a medios físicos tradicionales informativos como lo son los diversos soportes para prensa escrita (periódicos, revistas, magazines) se les añade un elemento de interactividad, a la vez que se extiende la imagen de otra manera bidimensional y estática. Así, no se pretende digitalizar la totalidad de la información, ni reemplazar el soporte comunicativo físico por una plataforma virtual, sino integrar el medio tradicional con formatos dinámicos disponibles en red. 


\section{Problemas y limitaciones de la Realidad Aumentada}

A pesar de las ventajas y conveniencias de emplear los sistemas de RA, se generan una serie de problemas y limitaciones derivados de la infraestructura tecnológica especificada. Las aplicaciones para usos en ambientes exteriores, traen como consecuencia que los dispositivos de visualización no estén debidamente protegidos frente a fenómenos climáticos adversos, o que estos dispositivos no respondan a los cambiantes niveles de luz natural. Además, pueden ser incómodos para el usuario, lo que repercute en un detrimento de su desempeño, aunque con los avances tecnológicos recientes en los dispositivos de telefonía móvil, eventualmente se están superando estas limitantes. La representación de los elementos virtuales puede presentar inconvenientes por sobrecarga visual para el usuario y tiempo de respuesta de la aplicación, que para construir muchos objetos requerirá del uso de más memoria (van Krevelen \& Poelman, 2010). Para que la RA cumpla su objetivo, el proceso de registro y alineamiento de los objetos virtuales con el espacio real tiene que ser preciso. El proceso de representar el espacio tridimensional en una pantalla de dos dimensiones, implica que pueden surgir fallos en el cálculo de las distancias donde deben estar ubicados los objetos (Azuma, 2001). Es fundamental sincronizar el movimiento de la cámara con los objetos virtuales para alinearlos correctamente con el entorno real; en aplicaciones para móviles, esto puede significar un alto costo en el procesamiento de las imágenes en tiempo real.

La aceptación social de este tipo de tecnologías, era un factor que amenazaba a la
RA, ya que dependía de la adaptación de los usuarios a los dispositivos de visualización y las formas de interacción; sin embargo, en los últimos años, la masificación de los dispositivos móviles y de aplicaciones de RA para los mismos, parece indicar una superación de este problema, surgiendo el desarrollo de aplicaciones de RA para teléfonos inteligentes como un mercado emergente y potencialmente productivo.

\section{Conclusiones}

Probablemente los dispositivos que más posibilidades ofrecen para desarrollar aplicaciones de RA en un futuro cercano sean los móviles (teléfonos celulares, tablets, consolas de videojuegos portátiles, etc), ya que muchos de ellos incorporan captura de imagen - uno de los requisitos técnicos para visualizar el ambiente real - y conexión a Internet, aspectos que unidos a su portabilidad y la creciente capacidad de cómputo, los hacen ideales para establecer procesos masivos de comunicación. Sin embargo, a pesar del carácter novedoso y llamativo de estas aplicaciones de realidad aumentada, encontramos una serie de desventajas en el proceso de masificación de las mismas, ya que en la mayoría de casos revisados se requiere, además de la posesión de los dispositivos, de un proceso de instalación de la aplicación y un periodo de aprendizaje de uso. Es así que, para superar el efectismo de ser considerada como una simple curiosidad y novedad tecnológica pasajera, la RA deberá asumir modelos de comunicación claros y concisos. Talvez el enfoque futuro de las aplicaciones de RA en el campo de los medios 
masivos de comunicación, está supeditado a su efectiva integración con las redes sociales para generar estructuras colaborativas de construcción de información. Adicionalmente, mientras muchas tendencias en el desarrollo de la realidad virtual apuntan a la generación de entornos figurativos, encontramos en la RA una oportunidad para explorar la concepción de elementos abstractos que se integren de manera espontánea a una realidad concreta.

\section{Referencias}

Aprenda a usar la realidad aumentada (n.d.). En El Colombiano. (7 de julio de 2012). Recuperado de:

http://www.elcolombiano.com/BancoConocimi ento/T/tecnologia_aprenda-a-usar-la-realidadaumentada-07072012/tecnologia_aprenda-ausar-la-realidad-aumentada-07072012.asp

Azuma, R.(1997). A Survey of Augmented Reality”. Presence, 6(4), 355-385.

Azuma, R., Baillot, Y., Behringer, S., Feiner, S., Julier, S \& MacIntyre, B.(2001). Recent Advances in Augmented Reality. IEEE Computer Graphics and Applications. 3, 34-47.

Basogain, X., Olabe, M., Espinosa, K., Rouèche, C. \& Olabe, J. C.(s.f.). Realidad Aumentada en la educación: una tecnología emergente.

Carracedo, J.(2011). Realidad aumentada: un nuevo paradigma en la educación superior. Actas del Congreso Iberoamericano Educación y Sociedad, CIEDUC.
Davivienda App. Recuperado de http://itunes.apple.com/us/app/davivienda/id52 $2110911 ? \mathrm{mt}=8$

Esteban, P., Restrepo, J., Trefftz, H., Jaramillo, J.\& Álvarez, N.(s.f.). La realidad aumentada: un espacio para la comprensión de cálculo en varias variables. EAFIT. Recuperado de http://www.iberomat.uji.es/carpeta/posters/ pedro_esteban.doc

Kato, H. \& Billinghurst, M. (1999). Marker tracking and HMD calibration for a video-based augmented reality conferencing system. Actas de International Workshop and Symposium on Augmented Reality, 4,.85-94.

Kesim, M. \& Ozarslan, Y. (2012). Augmented Reality in education: current technologies and the potential for education. Procedia - Social and Behavioral Sciences. 47, 297-302.

Knapp, M. (1982). La comunicación no verbal: el cuerpo y el entorno. Barcelona: Ediciones Paidós.

Levin, G. \& Lieberman, Z.(2004). "In situ speech visualization in real-time interactive installation and performance". En actas de 3rd international symposium on non-photorealistic animation and rendering.

Manovich, L. (2006). Abstraction and Complexity. Revista Kepes, 3(2),213-228.

Manual para usar realidad aumentada (n.d.). En El Tiempo. (2012). Recuperado de http://www.eltiempo.com/Multimedia/infografi a/realidadaumentada/ 
Ortiz, C. (2011). Realidad aumentada en medicina. Revista Colombiana de Cardiología, $18(1), 21-32$.

Quéau, P. (1992). Lo virtual: virtudes y vertigos. Barcelona: Paidós.

SoHo y la primera portada viva de Colombia (n.d.). En Revista SoHo. (2009). Recuperado de http:/www.soho.com.co/web/articulo/soho-y- la-primera-portada-viva-de-colombia/12412

van Krevelen,D.W.F. \& Poelman, R. (2010).

A survey of Augmented Reality technologies, applications and limitations". The International Journal of Virtual Reality. 9(2),1-20.

Zhou, F., Duh, H. B. \& Billinghurst, M. (2010). Trends in Augmented Reality tracking, interaction and display: a review of ten years of ISMAR”. Actas de ISMAR 2010. 